\title{
HANS GRÜNBERGER
}

\section{Wege zum Nächsten}

\author{
Luthers Vorstellungen vom Gemeinen Nutzen*
}

Luthers Vorstellungen vom Gemeinwohl sind in der politischen Ideengeschichte der Reformation bislang weitgehend unterbelichtet. Wird überhaupt eine ,lutherische' Konzeptualisierung von ,Gemeinnutz' thematisiert, so geschieht dies überwiegend unter rechtsgeschichtlichen und -theoretischen Gesichtspunkten, wie sie lutherische Juristen und Kameralisten verhandelt haben, weniger in einer Auseinandersetzung mit Luther selbst. ${ }^{1}$ Diese Vernachlässigungen erscheinen vor dem Hintergrund plausibel, daß Luther gemäß seinem Selbstverständnis als Theologe und Angehöriger der Universität und nicht als Inhaber eines politischen Amtes in die Bereiche politischer Ordnung interveniert hat, sei es als Berater, sei es in Predigt und Exegese und sei es in den weitverbreiteten Appellen und Vermahnungen. Sodann beruhen die Schwierigkeiten im Umgang mit den Schriften Luthers zur Politik, insbesondere seinen Darlegungen zu den zwei Bereichen mit ihren Regimentern geistlicher und weltlicher Natur und deren Komplementarität, vor allem auf ihrem parainetischen Charakter. Das heißt: In diesen Schriften ruht das Gewicht auf Mahnung und Warnung, und nicht notwendig auf Erklärung, da sie kein Gelehrtenpublikum zum Adressaten haben, sondern an die Hörer- und Leserschaft aller Stände und Ordnungen gerichtet sind, denen gegenüber vor allem Mißstände aufgezeigt werden sollen.

In diesen überwiegend auf deutsch verfaßten Schriften, welche in der Regel einem unmittelbaren Anlaß geschuldet sind, wird der Gemeinnutz vor allem in seinen zahllosen

- Bei der Zitation von Luthers Werken habe ich aufgrund der leichteren Erreichbarkeit zunächst die zuverlässigen Studienausgaben von Otto Clemen (Sigle $\mathrm{Cl}$.) und Hans-Ulrich Delius (Sigle MLStA) herangezogen. In den Fällen, in denen dort Texte fehlen, rekurriere ich auf die Weimarer Ausgabe (Sigle WA). Für Anregungen und Kritik, auch da, wo ich mich nicht habe anschließen können, danke ich Matthias Bohlender, Skadi Krause und Karsten Malowitz.

I Vgl. etwa die Ausführungen von Eckert 1976, die im ersten Kapitel ihrer Arbeit den topos des Gemeinnutzes im frühen Luthertum anhand der staatsrechtlichen Schrift Von dem Gemeinen nutze des Johannes Ferrarius 1533 verhandelt, den Reformator selbst, wie auch Philipp Melanchthon jedoch nicht in den Mittelpunkt ihrer Darstellung rückt. Zu den kommunalrechtlichen Aspekten des Gemeinen Nutzes vgl. den Beitrag von Peter Blickle (zu Ferrarius Abschnitt 2.3) sowie zur Gemeinwohltopik im Spätmittelalter und Frühen Neuzeit die Ausführungen von Thomas Simon beide in diesem Band. 
,Abwesenheiten " gegenüber der Omnipräsenz des Eigennutzes angesprochen. Damit aber wird zugleich eine politische und ökonomische Praxis, deren Essenzen auf den Diskurs von Intellektuellen im Dunst- und Gunstkreis der politischen Macht beschränkt blieb, auch den Machtunterworfenen zur Kenntnis gebracht. Sofern Luther Nähe und Zugang zu Machthabern hatte, nutzte er sie auch, um die Arcana an den Höfen derart aufzubrechen, daß er vor allem Defizite politischen und ökonomischen Handelns aufzeigt und dieses Wissen nicht allein den Adressaten überläßt. ${ }^{2}$ Besieht man sich aber darüber hinaus die Thematisierung des Politischen, wie Luther sie in seiner akademischen Profession als Exeget der heiligen Schriften - und hier zumal des Alten Testaments - betreibt, dann stellt sich der Eindruck ein, daß Gemeinnutz bei Luther zu einer Aufgabe im Sinne einer Zielorientierung aller Mitglieder der Gemeinde wird und der Reformator sich nicht auf ein Postulat an einen Landesherrn und -vater beschränkt. Sollte sich der Gemeinnutz jedoch erfahrbar einstellen, dann ist er als Gabe Gottes zu loben, zu der Menschen allenfalls zuarbeiten. Deshalb können sie eine gute res publica nicht im Sinne einer einfachen Zweck-/Zielprogrammatik politischen Handelns verwirklichen.

Im Sinne einer Annäherung an Luthers zu Unrecht wenig beachtete Vorstellung vom gemeinen Nutzen rekonstruiere ich nachfolgend zunächst das Verhältnis von Stand, Amt, Person in den drei Lebensbereichen der Schöpfung, da in ihnen und ihrem Verhältnis zueinander der gemeine Nutzen strukturell verankert ist (I.). Danach erörtere ich verschiedene Zugänge zu den Quellen des gemeinen Nutzen (Tugenden und Untugenden, der Dekalog) (II.). Anschließend diskutiere ich diese Zugänge mit Blick auf einen in ihnen enthaltenen Regulierungs- und Wissensbedarf und die netzwerkartige Bestimmung des gemeinen Nutzens (III.).

\section{Stand, Amt und Person: Handeln und Erleben in den drei Lebensbereichen der Schöpfungsordnung}

Zunächst ist festzuhalten, daß Luther eine Scheidung zwischen den Reichen Gottes/Christi als ,nicht von dieser Welt', und der diesseitigen Welt vornimmt. In Gottes Schöpfungsordnung auf Erden wiederum kommt es zur Unterscheidung von zunächst drei Ordnungen, mitunter auch ,Orden', ,Stände', ,Stiffte` oder schließlich ,hierarchiae` genannt: erstens der Bereich des ministerium verbi, primär der Bereich der Wortverkündigung im Sinne eines allgemeinen Priestertums, erst späterhin der Dienste in den Kirchengemeinden, respektive Berufe (ecclesia); zweitens der Bereich des Hauses, der Familie, respektive der Wirtschaft (oeconomia) mit seinen Ämtern und Berufen und schließlich drittens der Bereich des Gemeinwesens weltlicher Ordnung, politia, mit seinen Ämtern und Berufen. ${ }^{3}$ Die oeconomia und die politia sind weltliche Regimente im Sinne von Herrschaftsweisen in einem Herr-

2 Insoweit weicht Luthers Profil des Intellektuellen und seiner Wirksamkeit über die Grenzen gesetzter Exklusivität hinaus erheblich von demjenigen der Humanisten und juristischen Fürstenberater ab. Vgl. zum humanistischen Typus eines, staatsnahen`Intellektuellen Münkler 1998, S. 24-25.

3 Vgl. diese von Luther durchgehaltenen Einteilung der Stände in coram Deo gleichrangige Lebensund Aktionsbereiche Vom Abendmahl Christi, Bekenntnis $(1528)=$ Cl. 3:510:17-31. 
schafisbereich. ${ }^{4}$ Diese Ordnungen wiederum werden von Luther bezüglich der in ihnen agierenden und erlebenden Menschen zwei entscheidenden Relationen unterworfen: coram Deo versus coram hominibus - die Stellung des Einzelnen vor Gott und den Menschen. Die Unterscheidungen in zwei Reiche, respektive in die drei Ordnungen sind somit nicht dichotomisch gefaßt. Sie bündeln sich vielmehr wieder in den Personen, die diese Lebensbereiche gleichsam bevölkern. Denn zum einen kommt den Personen zu, in der Regel Mitglied aller drei ,göttlicher Stände' zu sein, die man als ,Teilsysteme' der Schöpfungsordnung fassen $\mathrm{kann}^{5}$ und zum anderen findet jeder Einzelne sich in einer gedoppelten Verantwortung: er steht mit seinem Stand allen anderen als Mitgliedern der Ordnungen untereinander gegenüber (coram hominibus) und er steht auch einzeln Gott gegenüber (coram Deo). ${ }^{6}$

Das Wirken in den jeweiligen Ordnungen erfährt seine institutionelle Einhegung durch das Amt. Die Ämter wiederum werden durch homines publici wahrgenommen. Hierfür tritt die Unterscheidung der homines publici versus homines privati inkraft: Den Amtleuten (homines publici) obliegt die Notwendigkeit, die weltliche Gerechtigkeit, mit der die Lehre der göttlichen Gerechtigkeit verteidigt werden kann und soll, durchzusetzen und damit auch zu strafen und zu richten. ${ }^{7}$ Gemäß dem Verständnis Luthers vom Amt hat der Mensch als homo publicus nicht sein eigenes Recht zu verteidigen, sondern das Recht anderer, das Recht des Nächsten. ${ }^{8}$ Mithin haben die Träger obrigkeitlicher Funktionen auf den Gemeinen Nutzen zu achten, wie aus ihrem Stand und Beruf deutlich wird: „Widderumb: die oberperson ist gesetzt das sie sol eine gemeine person sein und nicht allein für sich selbs, sol anhang haben der unterthanen und das schwerd fueren." Dieser ,gemeinen Person" als einer Trägerin ,öffentlicher Ämter ${ }^{\star}$ unterstehen als Untertanen immer eintzelne Personen, die jede für sich stehen:

„Denn wenn sich ein Fuerst zum Keyser keret, als zu seinem oeberherrn, so ist er nicht mehr Fuerst, sondern eine eintzelne person, ym gehorsam des keysers, wie alle andere, ein ieglicher für sich. Wenn er sich aber zu seinen unterthanen keret, als zu seinen unterthanen, so ist er so viel personen, so viel heupter er unter sich und an sich hangen hat."

Vgl. Bornkamm 1958, S. 36.

5 Vgl. zur prägnanten Zusammenführung der Teilordnungen in der Mehrfachmitgliedschaft der Personen: WATR 6: 266 = TR 6913: „Dreyerley göttlichen Stände sind von Gott geordnet, in welchen man mit Gott und gutem Gewissen seyn mag. Der erste ist der Hausstand; der ander das politische und weltliche Regiment; der dritte der Kirchen- oder Priesterstand nach den dreyen Personen der Dreyfaltigkeit. Erstlich mußt du im Hausregiment seyn entweder ein Vater oder Mutter, Kind, Knecht oder Magd. Zum Andern in einer Stadt oder lande ein Bürger oder Unterthan oder ein Oberkeit. Denn Gott hat die Menschen geschaffen,daß man sich freundlich und friedlich in Züchten und Ehren zusammen halten soll. Zum Dritten daß du in der Kirchen seyest entweder ein Pfarrherr, Caplan, Kirchener oder sonst derselben Diener, wenn du nur Gotteswort habst oder hörests. “

6 Vgl. Seils 1982, S. 33-34.

7 Sermo de duplici iustitia (1519)= MLStA 1: 226 Z 39-227 Z 1-5. Zur Unterscheidung der Gerechtigkeiten vgl. Grünberger 1999 mit weiteren Nachweisen.

8 Vgl. Bornkamm 1958, S. 42.

9 Ob Kriegsleute... $(1526)=$ Cl. 3:342:19-25. Nur in der letztlich entscheidenden Relation zu Gott, also coram deo sind, alle Menschen gleichermassen, einzeln' und haben als Einzelne sich zu verantworten. 
Die ,Stände', und hier interessierend oeconomia und politia, als Stiftung und Gabe Gottes haben Bestand und sind gleichsam in ,Wohlfahrt ${ }^{*}$ so es ihnen gelingt, zumindest die äußerliche Gerechtigkeit zu wahren. Deren Fundament wiederum bilden der Dekalog und seine jeweils regimentspezifischen Ausweitungen. ${ }^{10}$

Die hierin entfaltete Interpretation der zehn Gebote wird gemäß den Verhaltensrelationen Oberkeit - Untertan als politia, Herr - Knecht als oeconomia, sowie Mann - Frau als matrimonium aufgeschlüsselt und die Aufgaben der drei Stände dieses Lebens, der tres ordines vitae huius, werden entsprechend umschrieben: Sind die ersten beiden Gebote, (sie entsprechen der ersten Tafel des Gesetzes) der ecclesia zugeordnet, so dienen die Gebote 5 und 8 vornehmlich der Umschreibung der politia, während die Gebote 4 bis 7 und 9-10 die Sphäre der oeconomia erfassen. Als Verhaltensordnungen sind sie den ubiquitär geltenden Geboten der zweiten Tafel des Gesetzes unterworfen. Diese Gebote faßt Luther als Naturrecht und Summa aller äußerlichen Gerechtigkeit, Sie haben mithin Politik und Hausstand zu bestimmen, und an der Wahrung dieser Gebote haben die Amtsinhaber obrigkeitlicher Gewalt sich messen zu lassen. ${ }^{\text {" }}$

Demgegenüber hat Philipp Melanchthon zum Artikel XVI der Confessio Augustana von 1530, Policey und weltliches Regiment verhandelnd, eine Lesart eingeführt, die das Verhältnis der Oberkeiten zu den Untertanen im Sinne einer streng einzuhaltenden Institutionenhörigkeit definiert, wonach die Stände zunehmend als eine starre intransigente Ordnung gefasst werden, und nahegelegt wird, nicht nur das Amt, sondern auch die Träger des Amtes apriori als ,gut ${ }^{*}$, da von Gott eingesetzt, zu erachten. ${ }^{12}$ Desgleichen hat Melanchthon sowohl in seinem humanistischen Einführungstraktat zur Moralphilosophie als auch in seinem Aristoteleskommentar zur Politeia den Institutionencharakter jeglicher politischer Ordnung gegenüber den Personen als Machtträgern gestärkt und die Möglichkeiten aktiven Widerstands erheblich eingeschränkt, indem er den Stand der ecclesia in der Welt eindeutig unter die Fürsorge und das Regiment der politischen Ordnung stellt. Melanchthon weist die cura religionis, das heißt die Wahrung der ersten Tafel des Gesetzes, der politischen Ordnung zu, wie das im Übrigen die ebenfalls von Melanchthon entscheidend propagierte Visitationsordnung durch den Landesherrn erhellt. ${ }^{13}$ Die hieraus viel später auf der Grundlage der Konkordienformel von 1580 eta-

$10 \mathrm{Vgl}$. Iwand 1974, S. 231.

11 Vgl. hierzu Strohm 1983, S. 209.

12 Vgl. Confessio Augustana (1530) XVI: Von der Polizey und weltlichem Regiment / de rebus civilibus; in: BSLK, S. 70/71. und entsprechend Apologie der Konfession BSLK, S. 307-310; hierzu die Problematisierung bei Prien 1992, S. 167 und die grundlegende Erörterung bei Scharffenorth 1964, S. 145-155; hier S. 149-151.

13 Vgl. Melanchthon: Philosophiae moralis epitomes $1546=$ MStA III, S. 149ff.; sowie nachhaltig seine Commentarii in aliquot politicos libros Aristotelis (1531) in CR XVI: coll. 417-452; hier coll. $427 \mathrm{ff}$. und 449. Grundlegend die Unterschiede zwischen Melanchthon und Luther problematisierend Iwand 1966, S. 199-229, insbesondere S. 203ff.; 210-211; sowie Münkler 2000, S. 201f. In diesem Kontext ist auch die durch Melanchthon geforderte Stärkung des fürstlichen ,Summepiskopats ‘ zu sehen, das den Landesherrn auch über die Religion wachen und kontrollieren lässt. Vgl. dazu grundlegend Krumwiede 1967, sowie Grünberger 1997, S. 223-242; Grünberger 1999, S. 103-108. Demgegenüber haben Berman/Witte 1989, S. 1611-1635 die Verrechtlichung 
blierte Orthodoxie hat dann die nicht nur theoriegeschichtliche Konsequenz eines sowohl durch Apologeten wie auch durch Kritiker etablierten Konzepts einer starren Zwei-Reiche-Lehre gezogen, die dann wiederum Luther unterstellt wird. ${ }^{14}$

Während also bei Melanchthon in systematisierender Absicht die Institutionen im Vordergrund stehen, interessiert sich Luther vorrangig für die Akteure und also die Personen in den kirchlichen, politischen und (haus-) wirtschaftlichen Ordnungen. Diese Personenkreise sind in der Regel auch der Adressat seiner vermahnenden und gutachterlichen Schriften und zumindest was die Obrigkeiten angeht, sind diese nicht notwendig, ja sogar zumeist keine, Christpersonen: ${ }^{15}$

Die, weltbezogenen' Ordnungen wie auch das die Kirche betreffende ministerium verbi werden wohl eher durch eine Reihe analytisch getrennter ,Systemmitgliedschaften ${ }^{6}$ definiert: Das Amt, von Gott gesetzt, wird bei Luther durch Funktionsbeschreibungen definiert, die konstant gehalten werden: Das Amt ist gleichsam der Stand in Funktion ${ }^{16}$, dessen Beschreibungen immer dann aufgeführt werden, wenn es um den Nachweis geht, daß das Amt schlecht ausgefüllt wird oder wurde, wie etwa in den zahlreichen die politia betreffenden Fällen von Tyrannis. Diese Scheidung von Amt und Person hat Luther in seiner Auslegung des Magnificats $(1520 / 21)^{17}$ auf die Summe gebracht: „Merck aber, sie (= Maria) spricht nit, das er die stuel zubreche, ßondern wirfft die geweltigenn erauß. Spricht auch nit, Er lest die nydrigen hie nyden, Bondern erhebt sie. Denn die weil die welt steht, muß ubirkeit, regiment, gewalt, unnd die stule bleyben." 18

und damit die Institutionalisierung von Politik und Religion, wie sie in den systematischen Schriften Melanchthons angelegt sind, als eigenständige Leistung Melanchthons stark gemacht, habe diese doch ein in Staatlichkeit eingebettetes Luthertum erst ermöglicht.

$14 \mathrm{Vgl}$. Iwand 1966, S. 194 und 199.

15 Zu diesem Unterschied zwischen Luther und Melanchthon vgl. Münkler 1993, S. 644-645. Luthers Adressaten sind in der Regel politische Akteure (Fürsten, aber auch Stadtmagistrate, Bauernschaft, ,Kriegsleute* usw.) Melanchthon hingegen hat ein Interesse an einer Systematisierung von Dogmatik und - hier - politischer Ethik, mit dem er vor aristotelischem Hintergrund sein Konzept politischer Institutionen entfaltet. Luther hingegen lehnt Aristoteles nicht ab, sondern rezipiert ihn zunehmend stärker, liest ihn aber gleichsam, anwendungsbezogen" und damit auch elfenbeinturmsprengend: So stellt sich zum Beispiel vermittels seines häufigen Postulats, Billigkeit (epieikeia; Arist. Nikomach. Ethik $\varepsilon$ cap. 10) walten zu lassen, eine Anwendung aristotelischer Annahmen ein, wie etwa in seinen Ausführungen gegen die verschiedenen Beleihungsformen und des Wuchers. Hier unterscheidet Luther zwischen den Erfordernissen des Evangeliums, wonach Leihen gleich Schenken und restloses Hingeben des Eigentums sein solle. Auch Raub solle ohne Gegenwehr erduldet werden. Nach den Kriterien äußerlicher Gerechtigkeit auf Erden müsse jedoch in jedem Falle gegen Wucher bei erhöhtem Zins eingegriffen werden, während, bedingt durch Eigentumsverpflichtungen und sozialen Umständen, ein Zinssatz von $5 \%$ gemäß den Bedingungen der Billigkeit tolerabel sei. Vgl. im Einzelnen die Ausführungen bei Prien 1992, S. 123-140 et passim, zum Kriterium der Billigkeit S. 227-228.

16 Vgl. Maurer 1970, S. 7 unter Rekurs auf die Überschrift zur Haustafel in Luthers Kleinen Katechismus (1529): ,[...] fur allerley heilige orden und stende, dadurch dieselbigen als yhres ampts und diensts zu ermanen." (WA 30: I: 326: 17f.; BSLK 523).

17 Hier Lukas 1:52: „Er hat abgesetzt die gewaltigen von yhren stuhlen“.

${ }^{18}$ Cl. 2:174:36-175:1. 
Die Ämter selbst sind streng zu scheiden, da es ansonsten zu Überlastungen der Personen kommt, die dann ihrem Amt im Dienst am Nächsten nicht mehr gerecht werden:

„Und wenn sonst nichts were böses in den anderen und hohen Stenden, als da ist Geitz, Hoffart, Hass, Neid etc. So ist doch dis einige Laster böse gnug: Das sie Klug und Weise sein wöllen, da sie nicht sein sollen. Und jedermann geneigt anders zu tun, denn imp befolhen ist, und zu lassen, was ihm befohlen ist. Als wer im geistlichen Ampt ist, der will klug und thetig sein in weltlichem, und ist seiner weisheit hie kein ende. Widerumb, wer im weltlichen Ampt ist, dem wird das Heubt zu enge fur ubriger kunst, wie das geistliche Ampt zu regieren sey. “19

In diese Richtung ist vor allem auch der zweite Teil der Schrift Von weltlicher Obrigkeit, wie weit man ihr Gehorsam schuldig sei (1523) zu interpretieren. Hier zeigt Luther unter dem Titel „Wie weytt sich weltlich uberkeytt strecke "vor allem die Grenzen der ,Politik' und des politischen Amtshandelns auf, deren Verletzung, sei's durchs Papsttum, sei's durch die ,Kronen“ als Entgrenzung gedachter Systemgrenzen gelesen werden können: die Systemgrenzen einer Gemeinde als ecclesia und die einer Gemeine als politia:

„Denn meyn ungnedige herrn, Bapst und Bischoffe, sollten bischoffe seyn, unnd Gottis wortt predigen, das lassen sie, und sind weltliche fursten worden, und regirn mit gesetzen, die nur leyb und gutt betreffen. Feyn haben sie es umkeret, ynnerlich sollten sie regirn die seelen durch Gottis wortt, so regirn sie außwendig schloesser, stedt, land und leutt, und martern die seelen mit unseglicher moerderey. Also auch die welltlichen herrn, solten land und leutt regirn eußerlich, das lassen sie, Sie kunden nicht mehr, denn schinden und schaben, eyn zoll auff den andern, eyn zeyß $\mathrm{e}^{20}$ uber die andern setzen, da eyn bern, hie eyn wolff auslassen, Datßu kein recht trew noch wahrheytt bey yhn lassen funden werden, und handeln das reuber und buben zu viel were, und yhr welltlich regiment ja so tieff darnyder ligt, als der geystlichen Tyrannen regiment." 21

Personen sind vom Amt strikt zu unterscheiden, ${ }^{22}$ sie sind gleichsam kontingent. Das heißt: Überdauerung des Amtes im Falle eines Wechsels der Amtsinhaber. Die Personen haben sich dem Leistungsprofil des Amtes zu fügen, werden an diesem gemessen:

„Auffs erst ist der unterscheid für zu nehmen, das ein ander ding ist, Ampt und person, odder werck und thetter. Denn es kann wol ein ampt odder werck gut und recht sein an yhm selber, das doch boese und unrecht ist,, wenn die person odder thetter nicht gut odder recht ist, odder treibts nicht recht. ${ }^{\text {23 }}$

19 Die gantze heilige Schrifft deudsch (1545) Vorrede zu den Sprüchen Salomonis: vol.I: 1094: 18-29.

${ }^{20}$ zeyß $=$ Zins, respektive Steuer.

${ }^{21}$ Von weltlicher Obrigkeit $(1523)=\mathrm{Cl}$. 2:380:2-13; vgl. die scharfe Funktionsteilung zwischen geistlichen und weltlichen/politischen Ämtern Cl. 2:384:15-28; sowie Auslegung des 101. Psalms (1534-1535) = WA 51: 222: 28-223: 7.

22 Vgl. Luther, Kriegsleute: Cl. 3:318; Heerpredigt widder den Türken 1529/30=WA 30: II: 172: 2630.

${ }^{23}$ Ob Kriegsleute auch in seligem Stande sein können $(1526)=$ Cl. 3:318:37-319:2. Gleichfalls: „Es sind in allen Goetlichen ampten und stenden viel boeser menschen. Aber der stand ist und bleibt dennoch gut/wie hoch auch die menschen des misbrauchen. Man findet viel boeser weiber, viel falscher knecht, viel untrewer megde, viel schedlicher Amptleute und Rethe, Aber nichts deste weni- 
Dies betrifft schließlich die weitere Unterscheidung der Personen schlechthin: die Unterscheidung von Christperson und Weltperson ${ }^{24}$. Gilt für die Christperson ein Verhalten für sich gemäß der Bergpredigt und ihren Forderungen nach Feindesliebe und Vergebung; so ist die Weltperson - hier als Amtsperson - in ihrem Verhalten für andere an die Obliegenheiten des regierenden Standes gehalten; dies in Anlehnung an das Recht und die Pflicht zur Aufrechterhaltung einer äusseren Ordnung. Bindeglied von Christperson und Weltperson wird das Kriterium der Billigkeit - wider eine buchstabengetreue Gesetzlichkeit des summum ius summa iniuria - nach der sich auch die christliche Amtsperson zu orientieren habe. ${ }^{25}$ Träger und Mitglieder der Herrschaftsbereiche und Regimente sind immer die Personen als Weltperson und Christperson, in ihrer letztendlichen Verantwortung vor Gott, denn jede Christperson ist immer auch Weltperson, ist doch der Christ immer aufgefordert, auch Ämter im weltlichen Regiment zu übernehmen und sich hierfür die Bildung anzueignen, respektive seinen Söhnen eine entsprechende Ausbildung für ein politisches Amt zu ermöglichen. ${ }^{26}$ Indem schließlich Reiche und Regimente, wie auch die Stände immer mit Blick auf die sie ausfüllenden Personen zu sehen und focussiert sind, können sie nicht als dichotomisch geschieden gedacht werden, allein schon, weil die Reiche und ihre Regimente die Beziehungszusammenhänge konstituieren, in denen jegliche Person lebt. ${ }^{27}$ Die Person erscheint bei Luther im Zusammenhang seiner politischen Ethik jedoch weniger in ihrer durch einen freien Willen gekennzeichneten Individualität. Sie ist vielmehr, modern gesprochen, Focus eines Rollenkonfliktes von Amtperson und Christperson, der nur coram Deo, nicht aber vor den Menschen zu lösen ist. Insofern werden die getrennt gedachten Sphären der Reiche eines regnum mundi versus eines regnum Christi in der Person des Christenmenschen wieder zusammengeführt. ${ }^{28}$

Füllen die Amtsträger ihr Amt recht nach Maßgabe des Evangeliums und seinen Modifikationen durch das Naturrecht aus, erfüllen sie ihren Beruf. Der Beruf (vocatio)

ger, ist Frawen stand, Knechte und Magd stand, und alle ampt, gleich wol Gottes stifft werck und ordnung." Eine Predigt, daß man Kinder zur Schulen halten solle $(1530)=$ Cl. 4:171:9-14. Vgl. auch die Warnung vor Entgrenzungen der Scheidung von Ämtern und Berufen in den weltlichen Ordnungen im Sinne von Übergriffen: Vom Kriege widder die Türcken (1528)=WA 30: II: 112: 13-29.

${ }^{24}$ Vgl. Ebeling $1981^{4}$, S. 219-238; Duchrow 1970, S. 536ff.; Mau 1982, S. 17-18. Eckert 1973, S. 45 hingegen setzt Christperson mit ,Privatperson' mit Blick auf die Eigenverantwortlichkeit coram deo gleich, weshalb ihr der entscheidende Gegensatz Weltperson versus Christperson entgeht.

25 Vgl. diesbezüglich exemplarisch den Fürstenspiegel innerhalb der Auslegung des 101. Psalms = WA 51: 205: 28-206: 15 und mit Blick auf eine mögliche Bekämpfung des Wuchers: An die Pfarrherrn wider den Wucher zu predigen (1540) = WA 51: 352: 15-26, hier unter explizitem Rekurs auf die Diskussion der ,epieikeia ' und ihrer Voraussetzungen bei Aristoteles, Nikomachische Ethik Buch 5 ( $\varepsilon$ ) cap. 10.

${ }^{26} \mathrm{Vgl}$. Strohm 1983, S. 208; Prien 1992, S.159 unter Rekurs auf Eine Predigt, dass man Kinder in der Schulen halten solle $(1530)=$ WA 30: 2: 565: 10ff. $=$ Cl. 4:167:35-168:6; vgl. auch Vom Kriege widder die Türcken $(1528)=$ WA 30: II: 132: 7-20.

${ }^{27}$ Vgl. Bornkamm 1958, S. 30-31; hierzu Duchrow 1970, S. 525-527 und Prien 1992, S. 157-158, Anm. 68.

28 Vgl. Ebeling 1981, S. 227 ff.; Oberman 1986, S. 229ff.; Münkler 1993, S. $638 f$ f. 
entspricht einer Anforderung an die Person, ist mithin die personale Perspektive, der das Amt als institutionelle Perspektive zu korrespondieren hat. Damit gewinnt auch das Konstrukt der Larve (larva Dei) bei Luther eine politisch relevante Position. Über die Larven erscheinen Hausstand, politischer und geistlicher Stand koordiniert und damit auch ,gleichwertig ${ }^{29}{ }^{29}$ Larvae gibt es so nur in den drei Ständen des irdischen Reiches, in dem der Mensch sein Amt hat, wo er arbeitet und Werke gegenüber seinem Nächsten tut. Wie immer er seinen Beruf im Amte ausübt, der Mensch wird bei Luther über das Medium der Larve als Werkzeug in Gottes Hand gesehen. ${ }^{30}$ Die Larve, wie überdies die Person, ist mithin immer als ein noch nicht zum Begriff geronnenes Rollenanforderungsprofil zu denken, das im günstigsten Falle durch einen Mit- und Zuarbeiter Gottes (cooperator Dei) respektive einen Wundermann Gottes ${ }^{3 l}$ ausgefüllt wird, der selbst einer Berufung (vocatio) respektive einer Erweckung folgt. Von daher tritt bei Luther Berufung an die Stelle von Herkunft im Sinne eines Ständeprinzips. „Nicht die Geburt definiert den Beruf, sondern die vocatio, das Mandat Gottes. ${ }^{\prime 32}$ Mithin gestaltet sich der Zugang zu den Ämtern virtuell allen offen, da Eignung und Ausbildung vor Privileg und Geburt rangieren.

Wird nun mit dem Amt die Perspektive der jeweiligen Ordnung (,Stand', hierarchia) eingenommen, so tritt mit dem Beruf die motivationale Perspektive von Seiten der Person ins Blickfeld. Beruf (vocatio), figuriert für das Motiv, ein Amt ausführen zu wollen und zu glauben, es ausüben zu können. Dem Berufungsvorgang jedoch ist wesentlich, $\mathrm{da} ß$ in ihm ein Amt durch Dritte angetragen wird, er sich also nicht einer Eigenmächtigkeit des Berufenen verdankt. ${ }^{33}$ Ämter hingegen, seien sie geistlich und/oder politisch, haushälterisch, sind immer für den anderen, für den Nächsten auszuüben und nicht für sich. Das Bindeglied der Implementation von Amt und Beruf ist also die Orientierung am Nächsten und dessen Rechten, welche in beiden Fällen von Luther als Dienst definiert und qualifiziert wird. ${ }^{34}$

Darin mündet auch die Kritik Luthers am Mönchtum in seinem Traktat de votis monasticis (1521), sind doch das Mönchtum und sein Gelübde lediglich eine egoistische, aufs eigene Seelenheil fixierte Form, da es nicht für andere da sein, sondern nur für sich sein will. Aus der Orientierung am Nächsten, durch die jeder Beruf und jedes Amt gekennzeichnet ist, ergibt sich zudem das für alle Ordnungen geltende "Prinzip“ einer obligatio mutua. Exploriert werden muß jedoch weiterhin die sehr komplexe ,Verantwortungsethik': Verantwortet werden muß zunächst das Verhalten gegenüber der höheren Oberkeit und dann schließlich und vor allem Gott gegenüber. Also ist Verantwortung vom Amtsinhaber immer alleine zu tragen. Diese Eigenverantwortung ändert sich jedoch gegenüber Untergebenen: Gegenüber den Unterkeiten, respektive Un-

29 Vgl. Küppers 1959, S. 367-368.

30 Vgl. Wingren 1952, S. 92-93; Hermann 1960, S. 186-190; Mau 1982, S. 16.

${ }_{11}$ Vgl. hierzu Hermann 1960, S. 184-186; Ders. 1967, S. 174-177; Seils 1982, S. 32.

32 Strohm 1983, S. 211-212.

33 Vgl. Mau 1982, S. 21-22.

34 Vgl. Küppers 1959, S. 366; Scharffenorth 1964, S. 108; Prien 1992, S. 157-158. 
terpersonen, tritt die Einzelperson doch nunmehr als Träger eines Amtes auf, und kann so für sich institutionelle Gewalt beanspruchen. ${ }^{35}$

Aus den Verpflichtungen der Oberkeiten zur Wahrung der Zweiten Tafel des Gesetzes (Gebote 4-10 des Dekalogs) ergeben sich in Luthers Schriften eine Vielzahl an Varianten eines möglichen Widerstandes, die in eher entlegenen, lateinisch gehaltenen Traktaten, sermones und disputationes $^{36}$, ihren Niederschlag gefunden haben. Auf diese beziehen sich schließlich die Autoren um Nicolaus Amsdorff bei Abfassung ihres Magdeburger Bekenntnisses von 1550, woraus sich erhellt, daß ein Widerstandsrecht gemäß einer obligatio mutua für den Christen als Weltperson verpflichtend werden kann. Hier gilt, daß der Widerstand sich gegen die Person, die das obrigkeitliche Amt mißbraucht, richtet, nicht aber gegen das Institut der Obrigkeit, welche Gottes Setzung ist. Nicht zuletzt aber geht es zum einen um die Wahrung des allgemein verbindlich zu machenden Dekalogs, zum anderen aber um die Nichteinmischung der Politik in den Dienst am Wort. Denn auch das gilt: Die Mehrheit der Fürsten und der Oberkeiten sind verderbt und ein guter Fürst äuBerst selten: „Sihe zu letzt auch die hohen Fuersten stende an. Wo einer oder zween Christlich sind, die sind Wild bret im Himel, die andern alle bleiben helle brende mit dem Teuffel, und richten leid und unglueck gnug ueber die Christen“, wie Luther vor allem in seinen Schriften wider den Wucher nachhaltig betont. ${ }^{37}$

Die Untertanen als auch untergeordnete, sogenannte mittlere und untere Oberkeiten sind mithin verpflichtet, auf jeden Fall mit Wort und Rede Widerstand zu leisten, im Falle der Tyrannis gar mit Waffen. Allemal aber besteht die Verpflichtung - wohl für alle in der Gemeinde - nicht nur angesichts von Tyrannis und offensichtlichem Macht- und Amtsmißbrauch nicht zu schweigen, sondern öffentlich durch Gottes Wort zu ,strafen', wie Luther in seiner Politiklehre zu Psalm 82 auffordert:

„das nicht auffrhurissch ist die oeberkeit straffen, wo es geschicht nach der weise, die hier berurt stehet, nemlich das es durch Goettlich befolhen ampt und durch Gotts wort geschehe offentlich frey und redlich, Sondern es ist eine loebliche, edle seltsame tugent und ein sonderlich grosser gottesdienst.“

$35 \mathrm{Vgl}$. Ob Kriegsleute.... (1526) $=$ Cl. 3:334:3-336:5.

36 So zum Beispiel am entschiedensten die das Widerstandsrecht erörtende Circulardisputation zu Matth. 19:21 von 1539= WA 39: II: 34-91, welche sich gegen die weltliche Herrschaft des Papstes, dann aber auch gegen die kaiserliche Gewalt richtet. Siehe überdies auch ein von Kurfürst Johann 1530 angefordertes Gutachten, betreffend den Widerstand, in WAB 5: 258-261. Mithin kann die These Thomas A. Brady's, wonach Luther kein aktives Widerstandsrecht billige und nicht nur diesbezüglich ob seiner althergebrachten - old fashioned even in his own day - politischen Theorie eines Patriarchalismus als Wegbereiter des Absolutismus und autoritären Staates zu gelten habe, zumindest bezweifelt werden. Vgl. Brady 1986, S. 40-41.

37 Vgl. z.B. An die Pfarrherrn wider den Wucher zu predigen, Vermahnung 1540= WA 51: 406: 1011; Auslegung des 101. Psalms 1534/35 = WA 51:254: 11-12; siehe auch Von weltlicher Obrigkeit... $1523=\mathrm{Cl}$. 2:382:21-383:2, die generelle, Verderbtheit ${ }^{*}$ der Fürsten notierend unter denen ein ,frommer Fürst' ein gar seltsamer vogel sei. Vgl. Schempp 1972, S. 151-154. 
Denn nehme man die Pflicht, sich mit dem Wort gegen den Tyrannen sich zu erheben, nicht wahr, würde der Tyrann in seinem Wirken noch gestärkt. ${ }^{38}$ Diese Pflicht zum Widerstand betrifft alle Christen gerade im Wissen um die gemeinschaftsregulierenden Gebote des Dekalogs. Den einem Tyrannen Unterworfenen kommt hier die Qualität rechtmäßiger Obrigkeit zu: „Denn gleich wie die Oberkeit, welcher du ein glied bist, selbst widersteht, also gebeut sie dir auch widerstand zu thun vermoeg der andern Tafel, welcher du gehorsam zu sein schuldig bist." ${ }^{\text {"39 }}$ Verletzt nämlich die Obrigkeit ihre Verpflichtung gegenüber der Gemeinde, ist sie ebenso zu bestrafen, wie eine Gemeinde, die ihrerseits den Vertrag mit der Obrigkeit verletzt:

„Auff das die Gemeine gehorsam sey der oberkeit um Gottes willen, widderuemb die oberkeit recht und fride handhabe, auch um Gottes willen [...] Welchs teil aber das seine nicht thun will, sondern so die gemeine ungehorsam und die oberkeit mutwillig ist, sollen sie beide vor Gott des tods schueldig sein und gestrafft werden, die Gemeine durch die oberkeit, die oberkeit durch Gott... . .40

\section{Zugang zu den, Quellen' des Gemeinen Nutzens}

\section{Tugenden und Untugenden}

$4 m$ nächsten kommt man der Vorstellung Luthers vom Gemeinnutz aus der Perspektive Jer politia, wenn die drei Ordnungsziele angestrebt werden: (1) Schutz und Förderung ter Predigt des Evangeliums; d.h. Sicherung der ,Rahmenbedingungen' für die christlishe Gemeinde im Sinne der ersten drei Gebote. (2) Sicherung des Wohlstands und des jchutzes der Armen, Witwen und Waisen, da diese Tugend alle Werke der Gerechtigseit umfasse und begreife; und schließlich (3) Frieden zu halten, d.h. ungerechte Anvendung von Gewalt verhindern. Aus diesen drei Erfordernissen erwachse ein in Glück and Gottes Segen befindliches Gemeinwesen. ${ }^{41}$

\footnotetext{
${ }^{8}$ Vgl. Der 82. Psalm ausgelegt $1530=$ WA 31: I: 197: 29-34; hierzu Forck 1982, S. 67. Wie ein solcher Widerstand Form annehmen kann, hat Luther unter anderem in seinem Brief an die Fürsten zu Sachsen (= WA 15: 210-221) während des Bauernkriegs 1523, vor allem aber auch 1541 in seinem großen negativen Fürstenspiegel gegen Hans-Worst (Herzog Heinrich von BraunschweigWolfenbüttel) dargetan (=WA 51: 469-572). Daß ferner die (fürstlichen) Obrigkeiten sich nicht mit Hofschranzen und Speichelleckern umgeben sollten, sondern Kritik zu dulden, ja auf sie zu hören hätten, vgl. In XV Psalmos graduum Psalmus CXXVII (1532/1533)=WA 40: III: 265: 32-266: 16.

9 Circulardisputation über Matthäus 19:21 (1539) = WA 39:II 46: These \# 32 (deutsche Fassung). Vgl. Scharffenorth 1964, S. 139.

${ }^{0}$ Der 82. Psalm ausgelegt (1530) = WA 31:1: 193: 18-25; hierzu Elert 1953 II, S. 320.

1 Vgl. In XV Psalmos graduum XV; Psalmus CXXVIII= WA 40: III: 305: 21-24: „Primum est timor Dei; hic complectitur ea, quae primae tabulae sunt. Propter timorem Dei postea donat et conservat Deus domum et Oeconomiam, tandem propter Oeconomias recte institutas et in timore Dei administratas sequitur etiam tertia benedictio foelix scilicet Politia." Hier folge ich Prien 1992, S. 195-211. Wie Iwand 1974, S. 300f. zeigt, kann jedoch Luthers Auslegung des 82. Psalms = WA 31: 1 189218, insbesondere $196 \mathrm{f}$. als maßgeblich gelten; ebenso Psalm 127 in WA 40 III: 262-267, hier vornehmlich die Pflicht der Friedenssicherung.
} 
Der Katalog politischer Untugenden zeigt hingegen die Abwesenheit von Gemeinnutz auf und ist durch Grenzverletzungen der Teilbereiche von ecclesia, politia und oeconomia gekennzeichnet. ${ }^{42}$

Ich beschränke mich hier auf ein von Luther diagnostiziertes zentrales Laster politischer Regimente, den sogenannten Eigennutz, der in Luthers Schriften zur Politikgestaltung im Alten Testament breiten Raum einnimmt und damit ex negativo den Gemeinnutz erfasst, und sich in drei Untugenden manifestiert, die den eben benannten Tugenden und Bedingungen des Gemeinnutzes korrespondieren:

Die erste Untugend betrifft den Umgang seitens der Oberkeiten mit der ecclesia als Ort und Gemeinschaft der Wortverkündigung und deren Förderung, die sie unterlassen oder gar hintertreiben.

Die zweite Untugend betrifft den gleichsam selbstreflexiven Umgang der Oberkeiten mit ihrem eigenen Amt und ihren Pflichten gegenüber den ihnen Anempfohlenen: daß sie ihre Fürsorgepflicht außer Acht lassen und die Untertanen mit Unrecht und Gewalt drücken. Die dritte Untugend erkennt man im persönlichen Eigennutz der Amtsinhaber:

„das sie ym finstern wandeln und leben ynn solch Goettlichem Stand und ampt allein fuer sich selbst, gerade als hetten sie die Oberkeit dazu bekommen, das sie yhren nutz und ehre, yhre lust und mutwillen, yhren stoltz und pracht sollten suchen und treiben [...].“43

Dieser persönliche Eigennutz gestaltet sich facettenreich, und ist Ursache jeglicher Tyrannis, die grundsätzlich politia und oeconomia verstört, und deshalb ihren Sturz - aber nur deo volente - erforderlich macht. Das vorrangige peccatum politicum hat Luther in dem Hochmut und Egoismus von Tyrannen erblickt: „Mithin bin ich geradewegs der Meinung, daß (z.B.) Monarchien längeren Bestand gehabt hätten, wenn die Monarchen das eine Pronomen EGO aus ihrem Wortschatz und ihren Handlungen gestrichen hätten." $" 44$

Der Gemeinnutz wiederum erscheint seinerseits mit dem Entwurf eines (patrimonialen) Fürsorgestaats im Großen Katechismus beschrieben, wobei es Luther vornehmlich um ein Korrektiv zu den als Eigennutz gefaßten Sonderinteressen der zahlreichen eigenrechtlichen Stände geht, die im Übrigen die Anlässe zu seinen zahlreichen theolo-

${ }^{42}$ Thematisiert hat Luther diese Grenzverletzungen zunächst in gegenseitigen Übergriffen der Teilsysteme der Schöpfungsordnung, denn gegenseitige, gleichsam, systemgefährdende` Zugriffe hat Luther mehrfach beobachtet und zum Gegenstand von Schriften, Predigten und Disputationen gemacht. Vgl. exemplarisch seinen Vergleich von römischem Papsttum und der islamischen Herrschaftsordnung der Türken, das eher zugunsten des Letzteren ausgeht, in: Vom Kriege widder die Türken (1528) = WA 30: II: 107-148 und die Gefährdung von ecclesia und oeconomia zum Thema hat. Gleichsam akademisch werden die Systemgrenzverletzungen seitens der römischen Papstkirche in der Circulardisputation zu Matth. 19:2I (1539)=WA 39: II: 34-91 verhandelt.

${ }^{43}$ Der 82. Psalm ausgelegt (1530) = WA 31: I: 215:3-8; der gesamte, Untugendkatalog` ebenda: 214 : 20-215: 12.

${ }^{44}$ In XV Psalmos graduum XV; Psalmus CXXVII = WA 40: III: 225: 28-30: „Et ego plane in ea opinione sum Monarchias longe diutius fuisse duraturas, si Monarchae hoc unum pronomen EGO obmississent." Zur Idee des peccatum politicum auch Genesisvorlesung 1535-1545 = WA 42: 411: $10-16 ; 27-30$. 
gisch intendierten, beratenden Interventionen bieten. ${ }^{45}$ Den Verpflichtungen zu einem Gemeinnutz entsprechend hat Luther im Großen Katechismus zur Auslegung der vierten Bitte des Vater Unser das Scenario einer paternalistischen Fürsorgeordnung sowohl in politia als auch der haussständischen oeconomia umrissen und die frommen Fürsten aufgefordert, dem auch herrschaftssymbolisch Rechnung zu tragen:

„Daruemb moechte man billich ynn eines iglichen fromen Fürsten schild ein Brod setzen, fur ein lawen odder rawten kratz, odder auff die muentze fur das geprege schlagen, zu erynnern beide sie und die unterthanen, das wir durch yhr ampt schutz und friede haben, und on sie das liebe brod nicht essen noch behalten können. "46

Mit Blick auf eine Regimentenlehre aufschlussreicher und ,systematisch aufbereiteter erweisen sich jedoch die lateinischen Ausführungen zu den sogenannten Stufenpsalmen, hier Psalmen Salomonis 127 und $128,{ }^{47}$ die eine klare Unterscheidung von geistlichem, politischen und haushälterischen/familialen Bereichen bieten. In beiden Exegesen wird nach den Möglichkeiten des Strebens nach einem gemeinen Nutzen, der in der Orientierung am Nächsten verwirklicht werden soll, gesucht. Der Gemeine Nutzen wird von Luther immer und nur handlungsorientiert gesehen, sich ihm anzunähern geschieht nur durch eine Orientierung am personalen Nächsten in der Gemeinde. Von diesen beiden Polen, dem Nächsten und der Gemeinde her definiert sich Luthers implizite Gemeinwohlverpflichtung, der Amt und Beruf unterworfen sein sollten. ${ }^{48}$ Ein Gemeinwohl im Sinne eines politischen Zustandes, dessen Begriff noch nicht im Haushalt der politischen Semantik Luthers zugegen ist, scheint bei ihm nur vermittels einer wohlgeordneten res publica auf und ist, so es denn ausmachbar wird, Geschenk und Gabe Gottes, also nicht durch Menschen bewirkt, allenfalls durch ihre ,Zuarbeit' im Sinne eines $\mathrm{Ge}$ meinnutzes als einer auf den Anderen bezogenen Zielvorgabe. ${ }^{49}$ Als Wirken Gottes sieht Luther jedoch auch und vor allem, wenn die Menschen sich ihrer Zuarbeiter-Rolle nicht bewußt sind und deshalb trotz aller Vernunft, Wissen und Macht ihre in der Regel eigennützigen Ziele verfehlen. Die Heiden haben hierfür gerne zur Idee der Fortuna, also des Schicksals gegriffen, um den Mißerfolg erklärbar zu gestalten. ${ }^{50}$

${ }^{45} \mathrm{Zu}$ dem umfangreichen Katalog der Interventionen und ihrer Topoi vgl. die Zusammenstellung bei Elert 1953 II, S. 318-320.

46 Der grosse Katechismus (1529) „Die vierde bitte. Unser teglich brod gib uns heute" $=$ Cl. 4:71-73; hier 72:8-13. Vgl. hierzu Elert 1953 II, S. 411-412.

47 Vgl. WA 40: III: 202-269. Stufenpsalmen heißt: Lieder zu singen auf, Wallfahrten`. Die Zürcher Bibel setzt hierfür Wallfahrtslieder

48 Vgl. Strohm 1983, S. 212.

49 Vgl. In XV Psalmos graduum Psalmus CXXVII (1532/1533)= WA 40: III: 236: 29-36: „Sed redeo ad textum Psalmi, in quo videtis Oeconomiam et Politiam nobis commissas esse. Sed sic, ut sciamus nos instrumenta esse divinae maiestatis et organa seu cooperatores, non auctores, principia vel causas primas istarum divinarum rerum [...]."

50 Vgl. In XV Psalmos graduum Psalmus CXXVII (1532/1533) = WA 40: III: 242: 25-243: 30: „[Gentes - H.G.] Coacti igitur sunt dicere Fortunam dominari in rebus. [....] Post cum res aliter cederet, agnoscebant errorem et fortunae tribuebant omnia. Nos non debemus fortunae illa tribuere, nisi fortunam voces, quando sapientibus et potentibus ac mane surgentibus aliter cedunt sua consilia, quam praesumebant, sed iudicio Dei, qui praesumptionem sic ponit.“" Mit Luhmann läßt sich hier von ei- 
Während Psalm 127 als Vorlage dient, politia als Grundlage einer guten weltlichen Ordnung, welche zu errichten sei, zu deuten, werden für Psalm 128 die Perspektiven von Ehe und Hausstand erörtert, die komplementär zur politia angelegt sind und zudem den Rahmen für eine Darlegung der Arbeitsethik bereitstellen. Sie betonen den Vorrang von Ehe und Hausstand als Basis und Ausgangspunkt einer res publica und ,guten poli$c e y^{6}$. Der Vorrang ergibt sich schon daraus, daß Ehe und Hausstand komplexere Gemeinwesen erst ermöglichen. Sind jene gefährdet und verunsichert, schlägt sich doch diese Störung auf die komplexeren Ordnungen von Imperien und kommunalen Ordnungen nieder. Von daher sieht Luther in Ehe und Hausstand die Mittlerfunktion zwischen den übrigen Ordnungen der politia. ${ }^{51}$

Unter Psalm 127 Vers 2 „,Wo der HERR nicht die stad behuetet, so wachet der Wächter umbsonst" wird die Gemeinnutzperspektive bezogen auf politia und oeconomia. ${ }^{52}$ Was Salomo als Leben und Ort der Familie gekennzeichnet habe, begreife er, so Luther, heutigentages (= AD 1530) als Ehegemeinschaft (coniugium, matrimonium) oder auch als Haushaltung (oeconomia). Entsprechend redet er hier von einem Gemeinwesen (civitas (,Stad ), welches er als res publica einführt. Aus beiden Formationen, sowohl der oeconomia als auch der res publica, gibt es kein Entrinnen, weshalb es notwendig sei, über deren Zweck und Ziel zu erfahren und zu lernen, denn wir alle, ob Christen oder Nichtchristen, seien ,Mitglieder ${ }^{\star}$ einer politischen Ordnung und immer zugleich Mitglied einer Haushaltung, ${ }^{53}$ sei die politische Ordnung nun ein Königreich, ein Herzogtum, oder eine Bürgerschaft, sei diese res publica ein großes oder kleines Gemeinwesen. Für beide Ordnungen aber, die des Hauses und die des politischen Regiments gilt: Wenn die ,Lenkung und Leitung 'im häuslichen Bereich (gubernatio privata),wohl verfaßt und geordnet" ist, steht es auch gut um das Gemeinwesen schlechthin: Denn alle Haushaltung ist Ursache und Quelle, mithin die Grundlage eines Gemeinwesens. Ehestand und Haushaltungen hat Gott gestiftet und gebaut, die hieraus erwachsenden Gemeinwesen sind jedoch von Menschen einzurichten, werden aber vom Herrn gehütet. Denn auch gilt, daß, wo Vater und Mutter die Kinder hervorbringen und erziehen, gleichermaßen fehlen, ein Gemeinwesen keinen Bestand zu haben vermag. Erwächst doch aus dem (familialen) Hausverband gleichsam die ihm darin vermittelte Gemeinschaftsverpflichtung des einzelnen Menschen, für den es nicht gut sei, daß er alleine sei. Erst aus dem Hause, dem Haushalt, aber entsteht jegliches Gemeinwesen, welches für Luther doch nichts anderes ist als die Vielzahl

ner Ablösung von Kontingenzformeln in religiösen Ordnungen ausgehen, die Unerwartetes erklärbar und damit beherrschbar gestalten sollen. Was für Religion als Schicksal (als Göttin Fortuna) und schließlich als Gott fungiert, kann in der politia in der Zielvorgabe des Gemeinnutzes, respektive des Gemeinwohls gesehen werden; vgl. Luhmann 2000, S. 147-150, hier 148-149.

st Vgl. In XV Psalmos graduum Psalmus CXXVIII (1532/1533) = WA 40: III: 269: 22-32 (30-32): "Recte igitur dicitur Oeconomia fons Politiae, quod turbatis Oeconomiis non possunt constitui imperia nec civitates, sicut individuis sublatis neque species nec genus consistere potest.“

$52 \mathrm{Vgl}$. Iwand 1974, S. 305-306.

53 In XV Psalmos graduum XV; Psalmus $C X X V I I=$ WA 40: III: 210: 16-17 (Druckfassung): „Hoc vere est docere de Politia et Oeconomia, ostendere scilicet causam efficientem et finalem. Est autem eo magis necessaria haec doctrina, quod omnes vel in Politia, vel Oeconomia sumus. Etsi enim non sis maritus, tamen necesse est te esse in aliqua Oekonomiae parte." 
von Haushaltungen und Familien. ${ }^{54}$ Nun ist die Exegese zum 127. Psalm nach ihrem lateinischen Druck alsbald und gleich zweimal ins Deutsche übersetzt worden. Georg Maior hat den Kernsatz Oeconomia enim fons est Reipublicae 1536 so übersetzt: „denn die Haushaltung ist ein Ursprung, da von alle Polizeyen und Stadt Ordenung herkommen", während Caspar Hedio, der Straßburger Reformator, hingegen hier in seiner Übertragung von 1541 gleich eine Summe zieht: „Dann die haußhaltung eyn brunn ist des gemeynen nutzes. ${ }^{.65}$

\section{Die gemeinnützigen Verhaltens- und Kommunikationsregeln: Der Dekalog}

Einer seiner aufgezeichneten Universitätsveranstaltungen, einer Interpretation des Psalms 51 (1532/38), läßt sich entnehmen, worin Luther die Chancen und Grenzen einer Gemeinwohlperspektive unter den Voraussetzungen des weltlichen Regiments zu erkennen glaubt: Kraft der Barmherzigkeit und des Opfers Christi bedarf der Christ als Christperson keinerlei Institutionen und keinerlei Gesetze. Allein, im weltlichen Regimente sind Naturrecht und Gesetze in ihren Grenzen sinnvoll und nützlich. „So auch ist Recht und Gerechtigkeit eines Gemeinwesens in ihren begrenzten Zielen und Zwecken eine äußerst hilfreiche und gute Sache, damit Friede und Gemeinschaft auf wechselseitiger Basis bestehe unter den Menschen. ${ }^{(56}$ Der Fürst, respektive die Träger einer von Gott in Verantwortung gegenüber Gesetz und Evangelium gesetzten Oberkeit haben ihren Untertanen Schutz und Trutz zu gewähren, wie dies in Luthers als Fürstenspiegel zu lesenden Auslegungen des 82. und 101. Psalms zur Pflicht der Obrigkeit am Beispiele Davids deutlich gemacht wird. ${ }^{57}$ Entsprechend diesen Leistungen und Pflichten seitens des Fürsten haben die Untertanen der Obrigkeit im Gegenzug Gehorsam und Ehrerbietung zu leisten. Dabei ist es notwendig, die Aufgaben der Obrigkeit bezüglich ihres Wächteramtes, der custodia utri-

\footnotetext{
${ }^{54}$ Vgl. In XV Psalmos graduum XV: Psalmus CXXVII=WA 40: III: 219-221 (Druckversion): „Sicut supra domum appellavit rem familiarem et quod nos dicimus coniugium seu Oeconomiam, ita hic civitatem appellat Rempublicam, sive sit regnum, sive Ducatus, sive civitas, sive quaecumque Respublica, vel magna, vel parva. [...] Diserte autem dicit: Nisi Dominus custodierit civitatem, non dicit aedificaverit, sicut supra de domo dixit. Quia, cum gubernatio domestica et privata bene est constituta, tunc etiam bene habebit Respublica. Oeconomia enim fons est Reipublicae. Si enim desint pater et mater, uxor et maritus, qui progenerent sobolem et educent, non poterit consistere Respublica. Ex domo igitur propagatur civitas, quae nihil aliud est quam multae domus et familiae. Ex civitatibus fit Ducatus, ex ducatibus Regnum, quod illa omnia coniugit. Harum omnium Oeconomia fons est, e Deo creata in paradyso, ubi dixit: ,Non est bonum hominem esse solum, Item: Crescite et multiplicamini."

55 In XV Psalmos graduum XV; Psalmus CXXVII =WA 40: III: 219-220.

56 Enarratio Psalmi LI (1532/1538) = WA 40: II: 455: 39-41: „Sic iusticia Politica in suo fine est res suavissima et optima, ut constet pax et societas mutua inter homines".

57 Vgl. vor allem Auslegung des 101. Psalms (1530)=WA 51: 234: Z 12-25. Zu den beiden von ihm sogenannten Obrigkeitspsalmen 82 und 101, vgl. Bornkamm 1948, S. 11-14, sowie zu Psalm 101 als Vorgabe der Durchsetzung einer Kirchen- und allgemeinen Sittenzucht Grünberger 1999, S. 105-106.
} 
usque tabulae des Dekalogs, wie Philipp Melanchthon sie in seinen loci praecipui ${ }^{58}$ formuliert hat, festzulegen.

Die erste Tafel umfaßt die ersten drei Gebote des Dekalogs. Sie gilt der cura religionis im eigentlichen Sinne und bezieht sich auf Gott und seine Verehrung und den Umgang der christlichen Gemeinde mit ihm. Gegenstand der cura religionis ist die Sicherung der Verkündigung des Evangeliums und der Verwaltung der Sakramente. In dieser Tafel ist zugleich die Verkündigung der göttlichen Gerechtigkeit enthalten, die durch Werkgerechtigkeit nicht erreichbar ist und sich somit nur den Christen, der sanctorum communio, eröffnet.

Die zweite Tafel des Gesetzes hingegen gilt dem Verkehr, dem Umgang der Menschen untereinander und läßt folgende soziale Zuständigkeiten treffen: Diese zweite Tafel des Gesetzes, um eine erste Gliederung sichtbar zu machen, summiert die Verhaltensordnungen (a) gegenüber Einzelpersonen (5. Gebot; 6. Gebot), (b) gegenüber den Mitgliedern eines Gemeinwesens, sei dies die Mitgliedschaft im Hausstand und/oder einer politischen Ordnung (4, Gebot), (c) gegenüber den Mitgliedern einer Wirtschaftsordnung im Sinne von einzelnen Privatpersonen und ihren Eigentumsverhältnissen ( 7. Gebot), sowie (d) in der Relation von ,Kollektiven“ (z.B.Handelsgesellschaften; oikoi) zueinander (9.und 10. Gebot).

Betreffend eine sachliche Funktionszuweisung kann der Dekalog gleichfalls aufgeschlüsselt werden: Wird so dem status ecclesiasticus im Sinne einer Sorge um die Religion des Wortes Gottes das erste bis dritte Gebot zugewiesen, so erhält der status politicus im 5.(Tötungsverbot/Kriminalordnung) und 8. Gebot (Falsch Zeugnis; üble Nachrede, Kommmunikationsregeln) sein Fundament. Das vierte Gebot bedient gewissermaBen beide status: den des Hausstandes, als auch den der Politik, insoweit der Elternstand die primordiale Oberkeit schlechthin ist. Das vierte Gebot, welches als das erste der zweiten Tafel des Gesetzes gilt, stellt somit ein ordnungstiftendes Gelenk zwischen den beiden sozialen status, des Hausstandes und des politischen Gemeinwesens, dar. Dem status oeconomicus hingegen wird das 6. Gebot (Ehe- und Sittenzucht), die Gebote 7 und 9-10 (Diebstahl; Wirtschaftsordnung unter Kollektiven betreffend, negativ formuliert: Summe der Wirtschaftskriminalität, Steuerhinterziehung, Wucher) zugewiesen.

Die Gebote und Verbote der zweiten Tafel des Gesetzes haben die Qualität universal geltenden Naturrechts und sind für alle Menschen, ob Christ oder Nichtchrist, verbindlich, und „,auch sonst ynn aller menschen hertzen geschrieben“, wie Luther summarisch in Auslegung des dritten Glaubensartikels im Großen Katechismus festhält. ${ }^{59}$ Sie regeln die Ordnung äußerlicher Gerechtigkeit und werden oder wurden mitunter von Heiden, wie den griechischen Gesetzgebern, den gesetzestreuen römischen Kaisern, wie Augustus, oder gar den durch Disziplin in ihrer Binnenordnung gekennzeichneten Türken oftmals besser eingehalten als von Christen. ${ }^{60}$ Und so sind es vor allem die Teile, die

${ }^{58} \mathrm{MStA}$ II/1: loci praecipui (1559) expositio decalogi, hier: $314-315$ und 331-332. Vgl. auch oben Anmerkung 13.

$59 \mathrm{Vgl}$. Großer Katechismus $(1529)=\mathrm{Cl} .4: 60: 34-39$

${ }^{60}$ Vgl. exemplarisch den Katalog ,guter ${ }^{*}$ und ,äußerlich" gerechter heidnischer Herrscher in: Auslegung des 101. Psalms (1534-1535) = WA 51: 207: 21-36. sowie sekundär: Gerstenkorn (1956) in G. Wolf (Hg.) 1972, S. 77-79. von Loewenich (1966) in G. Wolf (Hg.), S. 439. 
der zweiten Tafel des Gesetzes gewidmet sind, welche entlang den Geboten 4-10 des Dekalogs die soziale Ordnung der christlichen Gemeinde definieren und die Vorgaben für die Obrigkeit hinsichtlich einer salus publica formulieren. Dabei kann in nahezu allen Traktaten Luthers, vor allem aber in seiner Bibelexegese beobachtet werden, daß die extensiven Erörterungen des vierten Gebotes die societas politica im engeren Sinne definieren. Dieses vierte Gebot, welches Liebe und Gehorsam den Eltern gegenüber vorschreibt, wird auf das politische Leben schlechthin übertragen. Entsprechend notiert Melanchthon: Das erste Gebot der zweiten Tafel des Gesetzes sagt nicht: Suche die Muße der Einsamkeit und deine Vergnügungen, schaffe Dir Müßiggang usw. Vielmehr spricht es: Ehre Vater und Mutter, es heiligt Reiche und Gehorsam. ${ }^{61}$ Entscheidend ist hier, daß die Kirchenordnungen lutherischer Observanz in Anlehnung an Luthers Interpretation des vierten Gebots in seinem Großen Katechismus vom Entwurf der Obrigkeit als einem Vaterstand ausgehen. ${ }^{62}$ Diese patrimoniale Verfassung der politischen Ordnung findet sich bei Luther durchgängig, bedarf aber einer knappen Einordnung ihrer Relation zu den drei Ständen: ecclesia, politia und oeconomia. Für Luther ist gemäß der Schöpfungsordnung die ecclesia primordial. Ecclesia aber darf in diesem Zusammenhang nun keineswegs als Kirche übersetzt werden, ist vielmehr eine Schöpfung des Wortes - creatura verbi - und so als Relation der (Christen-) Menschen zu Gott, vermittelt über das Wort und dessen Verkündigung, zu verstehen.

\section{Vom Aufkommen eines Regulierungs- und Wissensbedarfs}

Innerhalb einer Genealogie der Stände steht diese Relation am Anfang, wie Luther in seiner großen Genesisvorlesung ${ }^{63}$ darlegt: Zu ihr tritt mit Eva als zweiter Stand das matrimonium, die Ehe, und aus der Ehe entwickelt sich mit dem Auftrag Gottes, sich zu mehren, die Elternschaft als erste Obrigkeit, und mit dieser entsteht der Hausstand, die oeconomia, so daß ecclesia und oeconomia der politia vorausgingen. Für eine Regelungs- und Normierungsfunktion wie auch für eine hieraus sich ergebende Repressionsfunktion einer Obrigkeit ${ }^{64}$ war im sündenfreien Stande noch kein Bedarf: Adam und die Seinen lebten ursprünglich in absoluter Ruhe, und in dieser Ordnung vor dem Fall bewirkte ein Fingerzeig Adams mehr als nach dem Fall alle Schwerter, Straf- und Sicherheitsmaßnahmen zusammengenommen. Denn nach dem Fall Adams, und das heißt mit der Sünde, kommt der Bedarf an schneidender und brennender Medizin auf: Es bedarf

${ }^{61}$ MStA II/1, S. 333, Z 30-34: „Non enim dicit prima Lex (sc. secundae tabulae): quaere solitudinem, tuas voluptates, facito tibi otium, sed inquit: Honora patrem et matrem, sancit imperia et obedientiam."

62 Vgl. Großer Katechismus in: BSLK: 586-605 (596-599). Analog: MLStA 3: Von weltlicher Oberkeit Dritter Teyll =62-63 Z 10-15.

$63 \mathrm{Vgl}$. Genesisvorlesung 1535-1545 = WA 42: 79-80 zu Gen. 2: 15-17.

${ }^{64}$ Diese Differenzierung entnehme ich Münkler 2000, S. 201, der sich ihrer jedoch nur zur Unterscheidung der politischen Theoreme Melanchthons und Luthers bedient. Sie charakterisieren gleichwohl einen ,postlapsaren" Funktionsbedarf, der zur angenommenen Setzung von Obrigkeit als Amt und Stand führe. 
der politischen Ordnung und der äußerlichen Gesetze, um den Verbrechen, wie Raub und Mord, Betrug und Hinterziehung, aber auch der Lüge und dem falschen Zeugnis, nicht zu vergessen dem Wucher, Einhalt zu gebieten: Die politische Ordnung und ihre Ämter einschließlich des als notwendig erachteten Henkers gleichen in medizinischer Analogie einem Brenneisen und einer schrecklich bitteren Medizin, durch die schädlich kranke Glieder abgetrennt werden, damit alles Übrige gesunde. ${ }^{65}$

Hatte die Vorstellung einer Relation Gott - Mensch über das eine Wort paradiesisches Dasein ohne weitere Institutionen garantiert, so ist die jetzt gegebene Ordnung des Gemeinwesens auf vielerlei gleichrangige Stände, Ämter und Berufe angewiesen. Zudem verweist Luther auf Funktionsverluste ehedem stabil gedachter Institutionen. So ist etwa der Umstand zu beobachten, daß die Erziehung der jeweils nachfolgenden Generationen nicht ohne Leid und vor allem nur unter großen Mühen erfolgen kann. ${ }^{66}$ Deshalb müssen die Obrigkeiten darauf verpflichtet werden, Erziehung und Bildung für alle Stände nicht nur einzurichten, sondern auch zu garantieren. Hierfür muss der Friede im politischen Gemeinwesen gewährt sein, dessen Sicherung selbst eines Bildungsprogramms und seiner Durchführung bedarf. ${ }^{67}$ Mit dem Sündenfall sind auch ein ausführliches Wissens und viele Bücher notwendig geworden ${ }^{68}$, um die politische Ordnung anzuleiten: Neben den Theologen treten Juristen, Mediziner in Wort, Schrift und Handeln auf, um einer durch und durch verderbten Menschheit Wissen zu vermitteln. Gleichzeitig muss aber nun zwischen, gut ${ }^{\star}$ und, böse ${ }^{\star}$ laufend unterschieden werden, um die Adressaten des jeweiligen Wissens entsprechend zu unterrichten. Nicht zuletzt macht das Beispiel des Turmbaus zu Babel die Notwendigkeit von Bildung evident, wird doch Bildung konstitutiv für das politische Motiv der Friedenssicherung als Quelle des Gemeinwohls, welches nur auf gegenseitigem Verstehen beruhen kann: Wird die Sprachverwirrung infolge des babylonischen Turmbauprojekts dem Eigennutz und der Eigenliebe des Tyrannen Nimrod geschuldet, so kann die Verwirrung als folgende Strafe nur durch Erwerb von Sprachkenntnissen gemildert werden. Eine Konsequenz von gegenseitigem Nichtverstehen nämlich sind Konflikte und Krieg, wie Luther in seiner Exegese zu Kapitel 11 der Genesis erläutert: Zunächst habe nur eine erste einheitliche Sprachordnung bestanden, die als festes einigendes Band der menschlichen Gesellschaft und deren Eintracht zu werten sei, gemäß dem Diktum: ,Gleich und Gleich gesellt sich gern.' Entsprechend unterhalte und verkehre der Deutsche gerne mit seinesgleichen in seiner Sprache. Wo aber die Sprachen vielfältig und unterschiedlich seien, komme es nicht zu gedeihlichen Handelsbeziehungen. Folgenschwerer noch entstehe $\mathrm{Haß}$ in den

65 Genesisvorlesung 1535-1545 = WA 42:79: 14-19.

$66 \mathrm{Vgl}$. Genesisvorlesung 1535-1545=WA 42:79: 28-30.

67 Vgl. Eine Predigt, dass man solle Kinder zur Schule halten; $1530=$ Cl. 4:162-163. Friede im Gemeinwesen (pax politica) als Voraussetzung von Bildung als, gemeinwohlstiftender' Faktor vgl. In $X V$ Psalmos graduum XV; Psalmus $C X X V I I I=$ WA 40: III: 303: 25-29. Ein detailliertes sowohl an der Heiligen Schrift wie auch am Humanismus orientiertes Bildungsprogramm wird überdies von allen Reformatoren gefordert. Insonderheit finden sich die Argumente bei Melanchthon und in starker Übereinstimmung bei den gleichfalls stark humanistisch orientierten Auffassungen Calvins.

$68 \mathrm{Vgl}$. Genesisvorlesung (1535-1545), hier Kapitel 2: 16-17 = WA 42: 80: 15-16: ,[...] hodie librorum infinitus est numerus, in quibus instituuntur Theologi, lure consulti, Medici $[\ldots]^{\text {c.. }}$ 
Gefühlen bei den Angehörigen der Nationen, deren Sprache man sich nicht verstehe: „Also hasst der Franzose die Deutschen und verachtet sie und die Italiener hassen und verachten alle Nationen außer ihrer eigenen." ${ }^{69}$ Mithin ist es angezeigt, in Politik und Wirtschaft die Sprachbarrieren zu überwinden, erweist sich doch, daß aus jener Sprachverwirrung feindselige gespaltene Gefühle, unterschiedliche Sitten und Gebräuche, veränderte Intelligenz und Studienbedingungen erwachsen, die vor allem aber die Saat allen Übels legen: Die Folge der Sprachverwirrung, dem Eigennutz geschuldet, ist nämlich die Verstörung des Gemeinwesens und der (Haus-)Wirtschaft.

\section{Gemeinnutz in einem sozialen Netzwerk der Gegenseitigkeit?}

Die Ständeordnung, vor Luther eher hermetisch - stratifikatorisch - gedacht, birgt bei Luther in sich noch weitere Diversifikationen, so daß es neue Berufe und Ämter geben werde, sind doch Stände in wachsendem Maße unzelich zerteilet. ${ }^{70}$ In dieser Hinsicht kann von einem sozialen Netzwerk dreier Lebensbereiche die Rede sein, dem die Mitglieder gemäß ihrer Stände und Ordnungen zuzurechnen sind. ${ }^{71}$ Diese Ordnungen werden in ihrer Relation zu Gott und Menschen von Luther als gleichwertig erachtet und dargestellt, wodurch die traditionale altkirchliche (katholische) Hierarchienlehre durchbrochen wird, die vom Primat des geistlichen Standes ausgeht, dem die Stände des Adels und der arbeitenden Bevölkerung untergeordnet sind. ${ }^{72}$ Nicht nur kann die luthersche ,Drei-Ständelehre' als ein usus der Zwei-Reiche-Lehre gesehen werden, deren weltlichen Bereich sie abdeckt, und sie diesbezüglich ablöst. Sie durchbricht, wie Heiko Oberman notiert, „das Monopol der Priester und Mönche zugunsten des ,Gottes-Dienstes' eines jeden Berufs: ,Alles eitel heiligthum und heilig leben vor Gott" “" "73

Und so kann im Anschluss an Prien und Schwarz ein funktionaler Standesbegriff bei Luther angenommen werden: ${ }^{74}$ Die Stände weiten sich gemäß einem Funktionsbedarf aus: Der hier interessierende Stand der weltlichen Obrigkeit (politia/magistratus) reicht vom

69 Vgl. Genesisvorlesung (1535-45) hier Kap. 11 = WA 42: 411-413. (413: 3-15): „Hoc peccatum dicit Moses secutam poenam, divisionem linguarum, quae levis poena fuisse apparet, sed profecto horribilis est, si respicias ad gravissima incommoda, quae hanc linguarum divisionem sunt secuta. Nam primum linguae similitudo firmissimum vinculum est societatis humanae et concordiae. Nam hic quoque verum est, quod proverbio dicitur: ,similes similibus facile congregari` Germanus libenter cum sue gentis homine colloquitur et conversatur. Ubi autem linguae diversae sunt, ibi non solum nulla commercia intercedunt, sed etiam in animis odium nascitur eius gentis, cuius tu linguam non intelligis. Sic Gallus odit et contemnit Germanos, Itali oderunt et contemnunt prae se omnes Nationes. Apparet igitur ex ista linguarum divisione, dissociatos animos, mutatos mores, mutata ingenia et studia, ut vere eam appellare possis seminarium omnium malorum. Nam et Politiae et Oeconomiae turbationem excitavit."

70 Auslegung zu Matth. 18-24 (1537-1540) = WA 47: 452: 19-20. Zum Unterschied zwischen der Luther'schen Drei-Ständeordnung und dem altkirchlichen Ständeprinzip vgl. Schwarz 1984, S. 79.

71 Zu dem Konzept eines sozialen Netzwerks in Luthers Ständeordnung vgl. Brady 1986, S. 35.

72 Dies ist das Thema von Küppers 1959.

${ }^{73}$ So Oberman 1984, S. 28 unter Rekurs auf die oben, Anm. 3 zitierte Stelle in: Vom Abendmahl Christi, Bekenntnis (1528).

${ }^{74}$ Vgl. Prien 1992, S. 166 und Schwarz 1984, S. 83. 
Fürsten über Richter, Amtsleute, Kanzler und Schreiber bis hin zu den Bürgern als Mitgliedern von Stadtmagistraten, die alle Verantwortung haben. Insoweit erscheint dann auch der Fürst Kontrollen durch diese institutionalisierten und professionalisierten ,mittleren und unteren' Oberkeiten unterworfen und der Begriff der Oberkeit bezieht sich mithin nicht nur auf den Fürsten alleine, sondern auf eine Körperschaft herrschender und verwaltender Personen. ${ }^{75}$

Ähnlich ist die Ausweitung des Ehestandes zum Hausstand, zum status oekonomicus zu sehen, der nicht nur die Rechte, sondern auch die Pflichten der Eltern in ihrem Amte als Obrigkeit im Hausstand gegenüber Kindern, der Herren gegenüber ihren Knechten usw. umfasst.

„Das Kriterium für die Stände ist also, ob sie einen Dienst am Nächsten erfüllen“, ${ }^{76}$ den sie nach Maßgabe von gegenseitiger Hilfe einrichten sollten. Unter dieser Perspektive stehen dann auch die mehrfach wiederholten und erweiterten ,ökonomischen Schriften Luthers gegen den praktizierten Wucher, der ihn veranlasst, auf die Obrigkeit einzuwirken, in die Haus- und Wirtschaftsordnung ihres Herrschaftsgebietes einzugreifen. Ebenso hat mitunter die Pfarrgemeinde Funktionen der Obrigkeit zu übernehmen, wenn es um die Gestaltung und Verteilung eines Gemeinen Kastens geht, der, zur Versorgung von Witwen und Waisen gedacht, diese eigentlich der politischen Obrigkeit zugewiesene Funktion übernimmt. ${ }^{77}$ Und ebenso hat die Obrigkeit den Ehe- und Hausstand zunehmend von den Aufgaben der (Schul-) Bildung zu entlasten, was zum Thema der Schulreformschriften Luthers geworden ist.

Organisiert erscheint die politia analog zum Hausstand der oeconomia. In diesen beiden Bereichen des weltlichen Regiments hat die Dienstfunktion als Sorge für den Nächsten konstitutiv zu sein, wenn dem Gedanken des Gemeinwohls zum Durchbruch verholfen werden soll. Dies gilt sowohl für obrigkeitliche Aufgaben/Ämter wie auch für das Eigentumsverständnis in der Wirtschaftsethik und dem Wirken des Hausregiments. ${ }^{78}$ Die in Anlehnung an Aristoteles vorgenommene Gleichsetzung von Regierung und Hausstand hat Luther in die Metapher einer dreifachen Vaterschaft gefasst, unter der das vierte Gebot, den Elterngehorsam betreffend, Geltung habe: Dreierlei Väter werden demzufolge in diesem Gebot vorgestellt: Die Vaterschaft als natürliche Elternschaft, sodann vermittelt über Ehe und Familie das Amt des Hausvaters als der Familie wie auch dem Gesinde vorgeordnet, und schließlich die als ,Landesvater ${ }^{*}$ ge-

75 Vgl. Berman/Witte 1989, S. 1594-1595 und 1600.

${ }^{76}$ Prien 1992, S. 166.

77 Vgl. unter dem Gesichtspunkt wechselseitiger Hilfe der Teilordnungen ecclesia - oeconomia politia die interventionistischen Schriften Luthers: Sermon vom Wucher $(1520)=$ WA 6: 36-60; erneut erweitert in Von Kaufhandlung und Wucher $(1524)=$ Cl. 3:1-46; sodann der umfangreiche Traktat: Wider den Wucher zu Predigen- Vermahnung (1540) = WA 51: 325-424, worin Anleitungen gegeben werden, aus der Perspektive der ecclesia, den Wucher zu verwerfen, aber Modifikationen mit Blick auf eine sozial verträgliche Eigentums- und Sozialordnung zuzulassen. Die Ordnung eines gemeinen Kasten $(1523)=$ Cl. 2:404-423, verhandelnd eine Sozialordnung, unter anderem betreffend die Umwidmung des Ertrags aus konfiszierten Kirchen- und Klostergütern in einen ,Sozialhilfefonds` für die Gemeinde zu Leisnig.

78 Vgl. Prien 1992, S. 169-170. 
faßte Obrigkeit, ,welche (wie gesagt) alle ynn den vater stand gehoeret, und am aller weitesten umb sich greiffet, Denn hie ist nicht ein vater, sondern soviel mal vater, soviel er landsessen, buerger odder unterthane hat." ${ }^{\text {"79 }}$ Allein, dieser ,Landesvater', dem es durch Gottes Mandat obliegt, für die Untertanen zu sorgen und - wiederum vermittelt über den elterlichen Hausstand - Nahrung, Haus und Hof zu sichern, kann vielerlei Gestalt annehmen, nicht nur die des Fürsten, sondern zunehmend auch die Figuration einer vielschichtigen Obrigkeit (magistratus) ${ }^{80}$ wie sich zumal in den Möglichkeiten eines Widerstandes gegen mißbräuchliche Gewalt seitens der ,Oberkeiten' erweist.

In letzter Konsequenz aber stellt Luther auch die Vorstellung gemeinsamer Erfahrung und gemeinsamen Teilens von Freude und Leid als einer Vorraussetzung des Strebens nach Gemeinwohl und -nutz heraus:

„Wer ynn der gemeyne will seyn, der mus auch die last, fahr und schaden der gemeyne helffen tragen und leyden, ob ers gleich nicht verwirckt hat, sondern seyn nachbar, eben wie er des frids, nutzs, schutzs, gutts, freyheyt, und gemach der gemeyne geneust, ob er die selbigen gleich nicht erworben noch zu wegen gebracht hat (...) haben wyr gutts vom Herrn empfangen, warumb soellten wyr das boese auch nicht tragen?*81

Damit wird sichtbar, daß der Gemeinnutz als eine Zielvorgabe keine unmittelbare Leistung politischer Ordnung sein kann. Denn wird er nicht als Gnade Gottes empfunden, sondern als Eigenleistung, verwirkt er seinen Gemeinnutzcharakter und kehrt sich um in den Eigennutz, dem die politischen Ordnungen mit den Instrumenten der äußerlichen Gerechtigkeit gegensteuern sollten.

Sigla

BSLK= Bekenntnisschriften der evangelisch-lutherischen Kirche; Göttingen, 1930 u.ö.

$\mathrm{Cl} .=$ Luthers Werke in Auswahl vol. 1-8; Bonn am Rhein, 1912ff.; ab zweiter Auflage Berlin, hg. v. Otto Clemen. Zitation: Band: Seite: Zeile

$\mathrm{CR}=$ Corpus Reformatorum editio C.-G. Bretschneider und H.-E. Bindseil, Halle/Saale. Hier Melanchthon opera vol. XVI; 1850.

MLStA = Martin Luther Studienausgabe; hg. v. H.-U. Delius; Berlin 1987/1999; bislang 6 Bände. Zitation: Band: Seite: Zeile

MStA = Melanchthon Studienausgabe; editio R. Stupperich, Gütersloh, 1949ff ; 7 in 9 Bänden.

WA = D Martin Luthers Werke; Kritische Gesamtausgabe, Weimar, 1883 Zitation Band: Seite: Zeile. $W A B=W A$ Serie Briefwechsel

WATR $=$ WA Serie Tischreden.

79 Der Grosse Katechismus $(1529)=$ Cl. 4:24:29-33; hierzu Scharffenorth 1964, S. 71 sowie Brady 1986, S. 36-37.

${ }^{80}$ Berman/Witte 1989, S. 1599; Maurer 1970, S. 7; 24; Hermann 1967, S. 208-209; Scharffenorth 1964, S. 136-138.

${ }^{81}$ Ein Sendbrief von dem harten Büchlein wider die Bauern $1525=\mathrm{Cl} .3: 88: 10-17$. Hierzu vgl. Elert 1953 II, S. 426. 
Literatur

(o. Hrsg.) 1992 (11. A.), Die Bekenntnisschriften der evangelisch-lutherischen Kirche (1930), Göttingen. (Sigle BSLK)

Berman, H. J./Witte J. (1989), The Transformation of Western Legal Philosophy in Lutheran Germany, in: Southern California Law Review vol. 62, S. 1575-1660.

Bornkamm, H. (1948), Luther und das Alte Testament. Tübingen.

Bornkamm, H. (1958), Luthers Lehre von den zwei Reichen im Zusammenhang seiner Theologie; in: ARG 49, S. 26-49.

Brady, Th. A. jr. (1986), Luther and the State: The Reformer's Teaching in its Social Setting; in: Luther and the Modern State in Germany, hg. v. J. D. Tracy, Kirksville/Miss., S. 31-44.

Brecht, M. (19903), Martin Luther I: Sein Weg zur Reformation; Stuttgart.

Duchrow, U. (1970), Christenheit und Weltverantwortung, Stuttgart.

Ebeling, G. (1964), Luther, Einführung in sein Denken, Tübingen.

Eckert, B. (1976), Der Gedanke des Gemeinen Nutzen in der lutherischen Staatslehre des 16. und 17. Jahrhunderts, Diss. Phil. Univ. Frankfurt/Main.

Elert, W. $\left(1953^{2}\right)$, Morphologie des Luthertums II; München.

Forck, G. (1982), Die Aktualität der Zwei-Reiche-Lehre Luthers, in: Themen Luthers als Fragen der Kirche heute. Beiträge zur gegenwärtigen Lutherforschung, Berlin, S. 63-76.

Gerstenkorn, H. R. (1972), Von der Obrigkeit und von der Macht nach Martin Luther [1962], in: Luther und die Obrigkeit, hg. v. G. Wolf, Darmstadt, S. 77-103.

Grünberger, H. (1997), Institutionalisierung des protestantischen Sittendiskurses, in: Zeitschrift für Historische Forschung 24, S. 215-252.

Grünberger, H. (1999), Kirchenordnung zwischen göttlicher und äußerlicher Gerechtigkeit; in: Konzeptionen der Gerechtigkeit, hg. v. H. Münkler und M. Llanque, Baden-Baden, S. 101-124.

Haendler, G. (1979), Amt und Gemeinde bei Luther im Kontext der Kirchengeschichte, Stuttgart.

Hermann, R. (1960), Gesammelte Studien zur Theologie Luthers und der Reformation, Göttingen.

Hermann, R. (1967), Luthers Theologie (Gesammelte und nachgelassene Werke I, hg. v. H. Beintker), Göttingen.

Hermann, R. (1981), Studien zur Theologie Luthers und des Luthertums (Gesammelte und nachgelassene Werke II, hg. v. H. Beintker), Göttingen.

Iwand, H. J. (1966), Das Widerstandsrecht der Christen nach der Lehre der Reformatoren, in: Ders., Vorträge und Aufsätze, hg. v. K. G. Steck, München, S. 193-229.

Iwand, H. J. (1974), Luthers Theologie, hg. v. Joh. Haar, München.

Krumwiede, H. W. (1967), Zur Entstehung des landesherrlichen Kirchenregiments in Kursachsen und Braunschweig-Wolfenbüttel, Göttingen.

Küppers, J. (1959), Luthers Dreihierarchienlehre als Kritik der mittelalterlichen Gesellschaftsauffassung, in: Evangelische Theologie 8, S. 361-374.

Loewenich, W. von (1972), Luthers Stellung zur Obrigkeit [1966], in: Luther und die Obrigkeit, hg. v. G. Wolf, Darmstadt, S. 425-442.

Luhmann, N. (2000), Die Religion der Gesellschaft, Frankfurt/M.

Luther, M., Studienausgabe, hg. v. H.-U. Delius, Berlin 1979ff. (bislang 6 Bände) (Sigle MLStA).

Luther, M., Luthers Werke in Auswahl, hg. v. O. Clemen, Bonn $1912 \mathrm{ff}$., Reprint: Berlin, $1966^{6} \mathrm{ff}$. (Sigle $\mathrm{Cl}$.)

Luther, M., Die gantze heilige Schrifft deudsch (1545), hg. v. H. Volz, München/Darmstadt. 
Manns, P. (1984), Luthers Zwei-Reiche- und Drei-Stände-Lehre, in: Luther und die Politische Welt, hg. v. E. Iserloh und G. Müller, Wiesbaden, S. 3-26,

Mau, R. (1982), Beruf und Berufung bei Luther, in: Themen Luthers als Fragen der Kirche heute. Beiträge zur gegenwärtigen Lutherforschung, hg. v. J. Rogge und G. Schille, Berlin, S. 11-28.

Maurer, W. (1970), Luthers Lehre von den drei Hierarchien, München.

Melanchthon, Ph., Werke in Auswahl, Studienausgabe, hg. v. Robert Stupperich, Gütersloh, 1949ff. (Sigle MStA).

Münkler, H. (1993), Politisches Denken in der Zeit der Reformation, in: Pipers Handbuch der Politischen Ideen, Bd. 2: Von den Anfängen des Islams bis zur Reformation, hg. v. H. Münkler und I. Fetscher, München, S. 615-683.

Münkler, H. (1998), Einleitung zu Nationenbildung, in: Ders. u. a., Nationenbildung. Die Nationalisierung Europas im Diskurs humanistischer Intellektueller, Berlin, S. 13-28.

Münkler, H. (2000), Zwischen Humanismus und Reformation: Philipp Melanchthons und Martin Luthers Bild des Menschen und der politischen Ordnung, in: Geisteswissenschaftliche Dimensionen der Politik, hg. v. R. Kley und S. Möckli, Bern, S. 183-206.

Oberman, H. A. (1986), Die Reformation: Von Wittenberg nach Genf, Göttingen.

Oberman, H. A. (1984), Thesen zur Zwei-Reiche Lehre, in: Luther und die Politische Welt, S. 27-34.

Prien, H.-J. (1992), Luthers Wirtschaftsethik, Göttingen.

Scharffenorth, G. (1964), Römer 13 in der Geschichte des politischen Denkens. Ein Beitrag zur Klärung der politischen Traditionen in Deutschland seit dem 15. Jahrhundert, Diss. phil. RuprechtKarl-Universität Heidelberg.

Schempp, P. (1972), Ist Luthers Stellung zum Staat heute revisionsbedürftig? [1960], in: Luther und die Obrigkeit, hg. v. G. Wolf, Darmstadt, S 138-180.

Schmidt, K. D. (1972), Luthers Staatsauffassung [1967], in: Luther und die Obrigkeit, hg. v. G. Wolf, Darmstadt, S 181-195.

Schwarz, R. (1978), Luthers Lehre von den drei Ständen und die drei Dimensionen der Ethik, in: Lutherjahrbuch 45, Göttingen, S 15-34.

Schwarz, R. (1984), Ecclesia, oeconomia, politia. Sozialgeschichtliche und fundamentalethische Aspkte der protestantischen Drei Stände-Theorie, in: Protestantismus und Neuzeit, hg. v. H. Renz und Fr. W. Graf, Gütersloh, S. 78-88.

Seils, M. (1982), Die vernunftbezogene Welt. Ein Kapitel aus Luthers Theologie, in: Themen Luthers als Fragen der Kirche heute. Beiträge zur gegenwärtigen Lutherforschung, S. 29-40.

Strohm, Th. (1983), Luthers Wirtschafts- und Sozialethik, in: Leben und Werk Martin Luthers von 1526-1546, hg. v. H. Junghans, Göttingen, S. 205-223.

Wingren, G. (1952), Luthers Lehre vom Beruf, München. 